\title{
The optical dielectric function of polarizable liquids
}

Jan A. Leegwater and Shaul Mukamel

Department of Chemistry, University of Rochester, Rochester, New York 14627

(Received 23 February 1993; accepted 1 July 1993)

We report numerical simulations of the density of states and the frequency dependent dielectric function for optical frequencies of a polarizable Lennard-Jones atomic fluid from gas to liquid densities. Our simulations at high densities can be successfully interpreted in terms of fluctuations around a fcc solid reference model. The mean spherical approximation, which is based on a resummed density expansion, is shown to give reasonable predictions for intermediate densities but is shown to fail at liquid densities. We discuss the transition from the gas phase limit to the liquid phase theories, and show that for liquids nuclear motions do not strongly affect the line shape, whereas local field effects do.

\section{INTRODUCTION}

The optical properties of simple atomic liquids such as rare gases have been the subject of extensive experimental and theoretical studies. ${ }^{1}$ The static and dynamic properties of the electronic excitations and their dependence on liquid state structure have received considerablc attention. Recent interest is growing due to advances in ultrafast spectroscopic techniques as well as the study of small clusters which allow a direct and controllable study of effects of exciton confinement. ${ }^{2}$

Numerous theoretical methods have been employed in modeling dielectric fluctuations in condensed phases. These range from simple Drude oscillator models, selfconsistent procedures derived using path integral techniques, the mean spherical approximation (MSA) and numerical simulations. ${ }^{3-13}$ The MSA is one of the most widely used techniques, primarily due to its simplicity. It was first applied to dipole-dipole interactions by Wertheim, ${ }^{14}$ who obtained an analytical solution for the static dielectric constant $\epsilon(\omega=0)$. Wolynes suggested a direct generalization of the MSA equation to calculate the entire frequency dependence of $\epsilon(\omega) .{ }^{15}$ Recently Stratt and coworkers ${ }^{16}$ have calculated the absorption spectra and density of states for a hard sphere gas using the MSA. For the more physical model of dipole-dipole interaction their calculations covered a limited density range, up to about half of the density that corresponds to a liquid.

In this paper we report simulation results for the $a b$ sorption spectra and density of states for a Lennard-Jones fluid for a density range from the low density gas to a liquid near the triple point. We find that the MSA produces unphysical results for even moderate (dense gas) densities, for which we are not yet in a regime that can be qualified as liquid. Most liquid state theories use the ideal gas as a starting reference point. Our simulations do however compare favorably with results of an ideal solid and simulations for an Einstein solid. We propose a perturbation theory which incorporates disorder via small fluctuations around the solid as a simple way for modeling dielectric fluctuations in liquids.

\section{THE MACROSCOPIC POLARIZABILITY AND THE DIELECTRIC FUNCTION}

Our model consists of a collection of polarizable at$\mathrm{oms}^{17}$ and each atom is modeled as a four level system representing a ground $s$ state and a triply degenerate excited $p$ state. We hereafter denote this as the $s-p$ model. We assume the following Hamiltonian:

$$
H=\hbar \sum_{n i} \Omega B_{n i}^{\dagger} B_{n i}+\hat{J}+H_{\mathrm{nucl}}+H_{\mathrm{int}}
$$

The first two terms represent the electronic (dipoledipole) part of the Hamiltonian

$$
\hat{J}=\hbar \sum_{n<m, i j} J_{n i, m j}\left(r_{n m}\right)\left(B_{n i}^{\dagger} B_{m j}+B_{m j}^{\dagger} B_{n i}\right)
$$

In the summation, $n$ runs over the atoms that have transition energies $\Omega$, and $i$ runs over the internal degrees of freedom, $i=x, y, z . B^{\dagger}$ and $B$ are creation and annihilation operators for the $s-p$ system at site $n$. As long as we restrict ourselves to linear optical properties, these can be considered to have the standard Bose commutation relations

$$
\left[B_{n j}, B_{m j}^{\dagger}\right]=\delta_{n m} \delta_{i j} .
$$

In the present model we thus represent the system as a collection of Drude oscillators. ${ }^{3}$ For the linear optical susceptibilities discussed in this paper a classical theory produces identical results. Our treatment will be quantum mechanical with an eye towards the extension to nonlinear optical susceptibilities. The dipole-dipole interaction given here conserves the number of excitons (the HeitlerLondon approximation). It neglects terms of the form $B^{\dagger} B^{\dagger}$ and $B B$ whose contributions are of order $J / \Omega$ smaller than the terms considered, and can be safely neglected.

The couplings $J$ describe the dipole-dipole interatomic interaction

$$
\begin{aligned}
J_{n i, m j}\left(r_{n m}\right)= & -J\left[\frac{3 r_{n m, i} r_{n m, j}}{r_{n m}^{5}}-\delta_{i, j} \frac{1}{r_{n m}^{3}}\right] \\
& \times \exp \left(-\frac{r_{\mathrm{nm}}}{R}\right)\left(1-\delta_{n, m}\right),
\end{aligned}
$$


with $\delta$ the Kronecker delta. $J=\mu^{2}$, where $\mu$ is the transition dipole moment, is a parameter which determines the energy scale of the dipolar interactions. Alternatively, it can be related to the static polarizability $\alpha_{0}$ by $J=\alpha_{0} \Omega / 2$. The unit of $J$ is energy times volume. $R$ is a long distance cutoff that is introduced here to ensure that the Hamiltonian yields mathematically well defined results. Without a cutoff the expressions involving dipole sums such as Eq. (9) are conditionally convergent and they do not make unique predictions in the $k \rightarrow 0$ limit. At a suitable point in the calculation we take the limit $R \rightarrow \infty$. This procedure is discussed in detail in Ref. 17, where we have analyzed the dielectric properties of the model in the low density limit. $H_{\text {nucl }}$ is the Hamiltonian of the nuclear degrees of freedom

$$
H_{\text {nucl }}=\sum_{n} \frac{p_{n}^{2}}{2 m_{n}}+\sum_{n<m} V\left(r_{n m}\right),
$$

and $V$ represents the short range internuclear interactions other than the dipole-dipole forces which are included in $\hat{J}$. van der Waals forces should be included in $V$ as well since by invoking the Heitler-London approximation we have excluded them from the dipole part of the Hamiltonian. A typical form for $V$ is the Lennard-Jones potential used below. At low densities the contribution of $V$ is negligible, but this is no longer the case for liquid densities. Finally, $H_{\text {int }}$ represents the interaction of the system with the external electric field,

$$
H_{\text {int }}=-\sum_{n i} P_{n i} E_{n i},
$$

with the polarization operator

$$
P_{n i}=\mu_{n}\left(B_{n i}^{\dagger}+B_{n i}\right) \text {. }
$$

Our goal is to calculate the dielectric function

$$
\epsilon(k, \omega)=1+4 \pi \chi^{(1)}(k, \omega),
$$

with the linear susceptibility tensor

$$
\chi_{i j}^{(1)}(k, \omega)=\int_{0}^{\infty} d t\left\langle\left[P_{k, i}(0), P_{k, j}(t)\right]\right\rangle e^{i \omega t},
$$

where

$$
P_{k, i}=\frac{1}{\sqrt{N}} \sum_{n} P_{n} e^{i k \cdot r_{n}}
$$

The time evolution of $P$ in the Heisenberg picture is

$$
P(t)=\exp \left(\frac{i H_{0} t}{\hbar}\right) P \exp \left(\frac{-i H_{0} t}{\hbar}\right),
$$

where $H_{0}$ is the Hamiltonian $H$ without the interaction term $H_{\text {int }}$.

The numerical evaluation of the dipole correlation function meets some formidable difficultics. For short range interactions (decaying faster than $r^{-3}$ ) the relevant internuclear separations which contribute to the dipole correlation function are much shorter than the optical wavelength and we can safely make the long wavelength $(k \rightarrow 0)$ limit in our expressions. However, the dipole- dipole interactions are long range and contain a finite contribution of very far atoms separated by a distance larger than the optical wavelength. For this contribution the finite wavelength of light (finite $k$ ) needs to be carefully kept. Fortunately it is possible to use a procedure due to Ewald ${ }^{18}$ to separate the long range and short range contributions using a local field approximation for the long range contributions. This results in the Clausius-Mosotti relation for the long wavelength $(k \rightarrow 0)$ limit of $\epsilon(k, \omega), \epsilon(\omega)$,

$$
\frac{\epsilon(\omega)-1}{\epsilon(\omega)+2}=\frac{4 \pi}{3} n \alpha(\omega)
$$

where $\alpha(\omega)$ is defined as

$$
\alpha(\omega)=C(\omega)+C^{*}(-\omega),
$$

with the dipole correlation function

$$
C(\omega)=\int_{0}^{\infty} d t C(t) \exp (i \omega t)
$$

In the interaction picture with respect to the nuclear motions we have

$$
\begin{aligned}
C(t)= & \exp (-i \Omega t) \\
& \times \sum_{\gamma=x, y, z} \overline{\left\langle k=0, \gamma\left|\exp _{+}\left[-i \int_{0}^{t} d \tau \hat{J}(\tau)\right]\right| k=0, \gamma\right\rangle} .
\end{aligned}
$$

Here $\exp _{+}$is the positive time ordered exponential, and the overbar denotes average over all positions with respect to the equilibrium distribution of particles. In Eq. (15) we have introduced a basis set of exciton states in $k$ space

$$
|k \gamma\rangle=N^{-1 / 2} \sum_{n} \exp \left(i k \cdot r_{n}\right)|n \gamma\rangle,
$$

with $|n \gamma\rangle \equiv B_{n \gamma}^{\dagger}|g\rangle$ denoting the state in which atom $n$ is excited along the $\gamma$ direction. $\hat{J}(t)$ is the operator $\hat{J}$ in the interaction picture with respect to the nuclear Hamiltonian

$$
\hat{J}(t)=\exp \left(\frac{i}{\hbar} H_{\text {nucl }} t\right) \hat{J} \exp \left(-\frac{i}{\hbar} H_{\text {nucl }} t\right) .
$$

Using the Clausius-Mosotti procedure we can pretend that the sample is smaller than the wavelength of light. We may then set $k=0$ and calculate the polarizability $\alpha$. Subsequently Eq. (12) corrects for this approximation and applies to macroscopic systems which are much larger than the optical wavelength. Equation (15) is our basic formal expression and is the starting point for the present article.

\section{THE STATIC LIMIT: NUMERICAL SIMULATIONS AND COMPARISON WITH THE MSA}

We have recently studied the optical response of the present model at low (gas phase) densities. ${ }^{17}$ In this limit the frequency dispersion of the dielectric function has a roughly equal contribution from static (inhomogeneous) fluctuations in density and from dynamical collisions (homogeneous), regardless of the nuclear mass and temperature. It is commonly believed that spectral line shapes in liquids are inhomogeneously broadened, ${ }^{3-11}$ and can there- 
fore be calculated by the complete neglect of nuclear motions (the static limit). In this and the next section we calculate the dielectric function in the static approximation, assuming infinite nuclear mass. In Sec. V we shall estimate the effects of nuclear motion and examine the applicability of the static limit.

When nuclear motions are neglected we set $H_{\text {nucl }}=0$. The time ordered exponential becomes an ordinary exponential and the Fourier transform Eq. (14) can be performed resulting in

$$
C(\omega)=\frac{\mu^{2}}{3 \pi} \sum_{\gamma=x, y, z} \overline{\left\langle k=0, \gamma\left|\frac{1}{\omega-\Omega-\hat{J}+i 0^{+}}\right| k=0, \gamma\right\rangle},
$$

where $\hat{J}$ is the matrix whose elements are $J_{n i, m j}$ for a given realization of particles. The exact calculation of the dielectric function in the static limit for a system of $N$ atoms requires the diagonalization of a $3 N \times 3 N$ matrix. This is a much simpler problem compared with evaluating the time ordcred cxponential.

There are significant advances in the theory of random matrices which allow to draw some general conclusions regarding the distribution of eigenfunctions and the statistics of energy spacings. ${ }^{19}$ However these results are not directly applicable to the present model since the random matrix elements have diverging moments.

Another important quantity that can be calculated using the same matrix is the density of states

$$
\rho_{D}(\omega)=\frac{1}{3 \pi N} \operatorname{Im} \sum_{k, \gamma=x, y, z} \overline{\left\langle k, \gamma\left|\frac{1}{\omega-\Omega-\hat{J}-i 0^{+}}\right| k, \gamma\right\rangle .}
$$

We shall calculate it as well, and use it in the analysis of the dielectric function.

We have simulated the polarizability in the static limit. By introducing the cutoff $R, \hat{J}$ represents only the short range part of the dielectric interaction. Yet it is still very long range compared to box sizes attainable in simulations. We therefore treated it using periodic boundary conditions. The limit $R \rightarrow \infty$ can then be taken using the Ewald summation by replacing the dipole-dipole interaction by an effective one, ${ }^{18}$

$$
J_{i j}^{(\text {eff) }}(r) \equiv \lim _{R \rightarrow \infty} \sum_{r^{\prime}} \exp \left(-\frac{r+r^{\prime}}{R}\right) J_{i, j}\left(r+r^{\prime}\right),
$$

where $r^{\prime}$ runs over the simple cubic direct lattice. This limit can be taken analytically, resulting in a rapidly converging series expression for $J^{(\mathrm{eff})} \cdot{ }^{18}$ In this approximation the large distance contribution of the dipole-dipole interaction is partly taken into account by the summation over the periodic replicas, the remaining large distance effects are taken care of by the Clausius-Mosotti formula. By imposing periodic boundary conditions we map the system onto a lattice with many particles in a unit cell.

In the calculations reported below we focus on the imaginary part of the polarizability $\alpha$ and the dielectric function $\epsilon$ which are directly responsible for the linear ab-

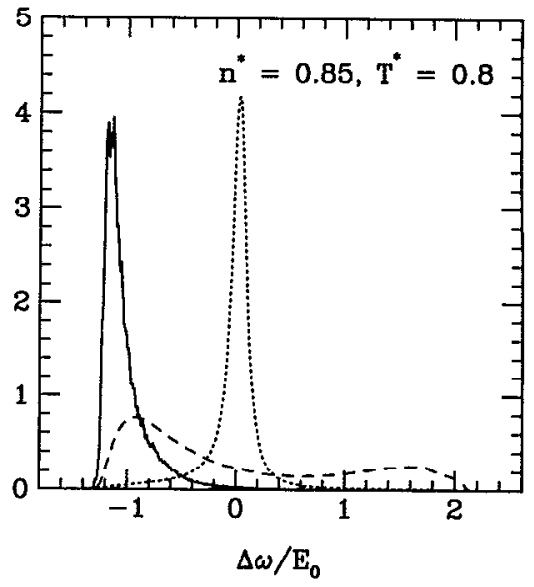

FIG. 1. The linear (polarizability) absorption spectrum $\epsilon^{\prime \prime}(\omega)$ (solid), $\bar{\alpha}(\omega)^{\prime \prime}$ (dotted), and the density of states $\bar{\rho}_{d}(\omega)$ (dashed) for a LennardJones liquid. This simulation is for 256 particles and nuclear motion is ignored. The isolated molecule transition frequency is at $\Delta \omega=0$. Compared to the low density results of Fig. 2, the spectra are narrower (in units of $E_{0}$ ) due to the atomic core repulsion.

sorption. The real parts can be obtained through the Kramers-Kronig relation. We adopt the common notation

$$
\alpha(\omega) \equiv \alpha^{\prime}(\omega)+i \alpha^{\prime \prime}(\omega)
$$

and

$$
\epsilon(\omega) \equiv \epsilon^{\prime}(\omega)+i \epsilon^{\prime \prime}(\omega) .
$$

We further introduce the parameter

$$
E_{0} \equiv \frac{4 \pi}{3} n J=\frac{2 \pi}{3} n \alpha_{0} \Omega,
$$

which represents the energy scale of the dielectric fluctuations, $n \equiv N / V$ being the atomic number density. In the following we express energies in terms of $E_{0}$. We present our results in dimensionless units, so

$$
\bar{\alpha}^{\prime \prime}(\omega)=\frac{\alpha^{\prime \prime}(\omega) E_{0}}{J},
$$

and

$$
\bar{\rho}_{D}(\omega)=\rho_{D}(\omega) E_{0} .
$$

In the simulations we used a molecular dynamic (MD) program to generate liquid state configurations for $N=256$ particles, then the matrix $\hat{J}$ was calculated and diagonalized using a library routine. The density of states and absorption spectrum was averaged over 250 configurations. The particles interact through the Lennard-Jones potential $V(r)=4 \epsilon_{\mathrm{LJ}}\left[\left(r / \sigma_{\mathrm{LJ}}\right)^{-12}-\left(r / \sigma_{\mathrm{LJ}}\right)^{-6}\right]$. The density is reported in dimensionless units, $n^{*}=n \sigma_{\mathrm{LJ}}^{3}$, and the reduced temperature is $T^{*}=k_{B} T / \epsilon_{\mathrm{LJ}}$.

In Fig. 1 we have plotted simulation results for the polarizability $\bar{\alpha}^{\prime \prime}$, the absorption spectrum $\epsilon^{\prime \prime}$, and the density of states for a Lennard-Jones liquid. For comparison we display the same quantities calculated at a very low 


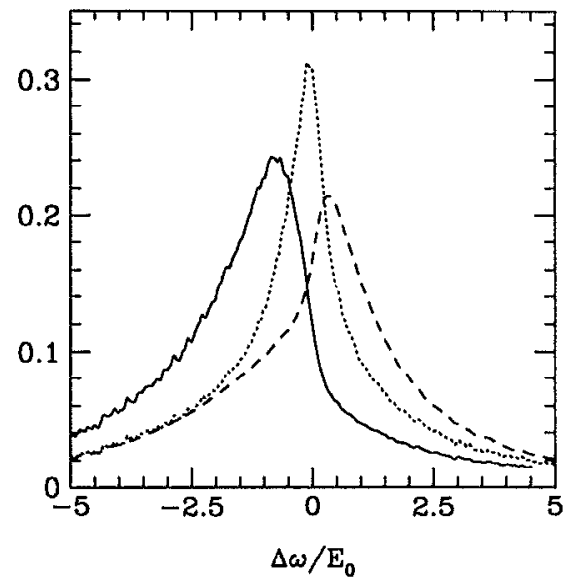

FIG. 2. Same as Fig. 1, but now the zero density limit is displayed. The energy is in units of $E_{0}=4 \pi n J / 3$. The low density limit is discussed in Ref. 17.

density in Fig. $2 .{ }^{17}$ At low density the present model tends to a limiting form that is independent on density apart from the energy scale $E_{0}$.

The following exact sum rules follow from the property of the dipole-dipole interaction $\langle k=0|J| k=0\rangle=0$,

$$
\int d \omega \omega \bar{\alpha}^{\prime \prime}(\omega)=\Omega
$$

and

$$
\int d \omega \omega \epsilon^{\prime \prime}(\omega)=\Omega-E_{0}
$$

For a liquid the absorption is strongest near the density of states band edge, the spectrum is asymmetric, and the sum rule then predicts that the maximum is shifted more than just $E_{0}$. This feature is apparent in the simulations, presented in Fig. 1.

Many features in the liquid are completely different from their low density counterparts. The high energy tails are completely absent due to the repulsion of the core. The density of states for a liquid is very different from the absorption spectrum, while for a gas at least in the tails they are the same. Local field effects, i.e., the difference between $\alpha^{\prime \prime}$ and $\epsilon^{\prime \prime}$, are much more important for a liquid, causing a substantial asymmetry of the absorption.

In Figs. 3-6 we report our simulation results at several densities and compare them with the predictions of the MSA. The MSA is reasonable at intermediate density $n^{*}=0.3$ (Fig. 4), which is about the critical density. Here the system is a dense fluid. At all other densities the MSA produces less than satisfactory results. Obviously the MSA, which is a theory based on a resummed density expansion does not produce an adequate framework for the interpretation of dielectric fluctuations. It should be added however that the MSA reproduces the exact first moment of the density of states. ${ }^{12}$ In the next section we show that a fcc solid does provide an adequate reference for analyzing these results.

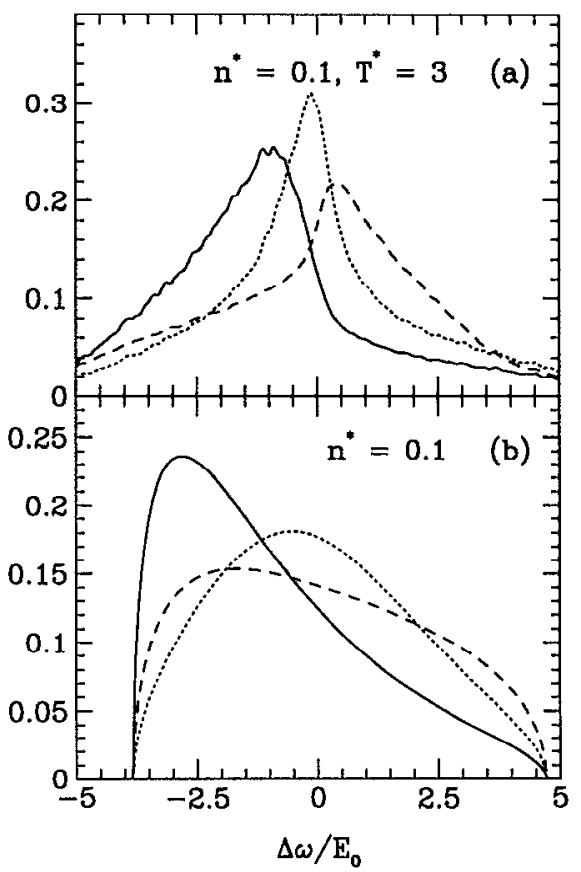

FIG. 3. Same as Fig. 1. Simulations results for a LJ gas (a) compared to the MSA for a hard sphere fluid (b) at a similar density. At this density we deal with a slightly dense gas. At this density the simulated spectrum is (still) similar to the low density spectra, Fig. 2.

\section{INTERPRETATION OF THE STATIC SIMULATIONS USING A FCC SOLID REFERENCE}

To gain further insight let us consider a regular lattice of atoms. In this case the isolated atom line is shifted by an energy which is the Fourier transform of $J$. As optical

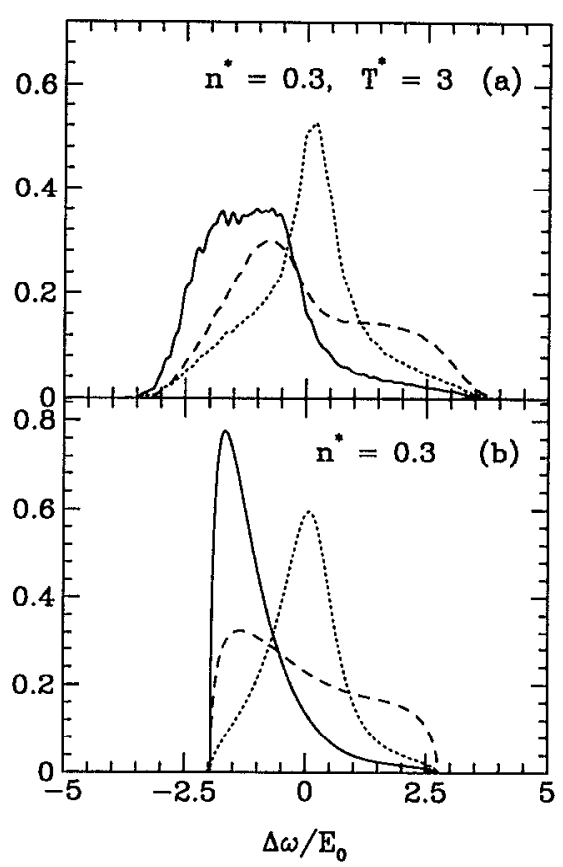

FIG. 4. Same as Fig. 1. Simulations results for a $\mathrm{LJ}$ gas (a) compared to the MSA for a hard sphere fluid (b) at a similar density. 


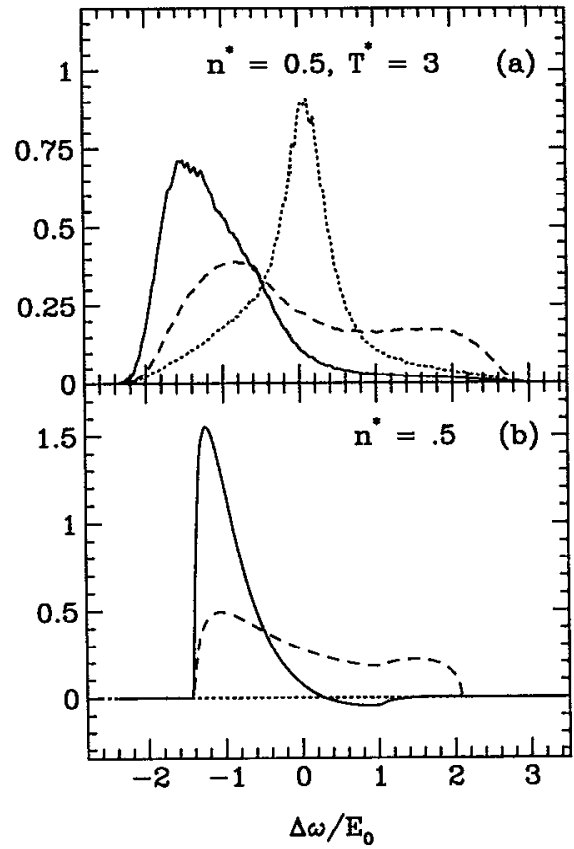

FIG. 5. Same as Fig. 1. Simulations results for a $\mathrm{LJ}$ gas (a) compared to the MSA for a hard sphere fluid (b) at a similar density. At this density we deal with a moderately dense gas. The MSA produces reasonable results for the density of states, but unphysical results for the absorption coefficient. $\alpha$ " becomes negative as well (not shown).

wavelengths typically are much larger than the interparticle separation we consider the $k \rightarrow 0$ limit where we have

$$
J(k)=E_{0}(3 \hat{k} \hat{k}-1) .
$$

The collective many atoms states (elementary excitations) for a lattice are called excitons. ${ }^{20}$ The longitudinal (trans-

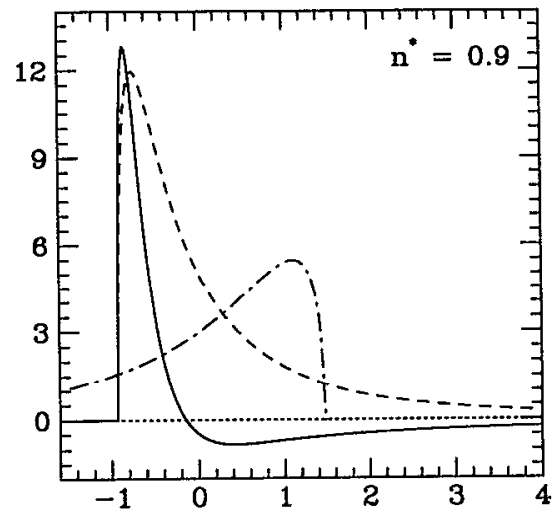

FIG. 6. MSA for a hard sphere fluid at liquid state density. The solid line is the absorption spectrum, which becomes negative and therefore is unphysical. The dashed line is the density of states that follows from one solution the MSA equation, the dot-dashed line is a different solution. The continuation at the crossing point follows from the real parts of the Green function. There is no way to choose the "most physical" solution and have a continuous absorption spectrum. The failure of the MSA at densities which are comparable to liquid densities rules out its role as a reference theory.

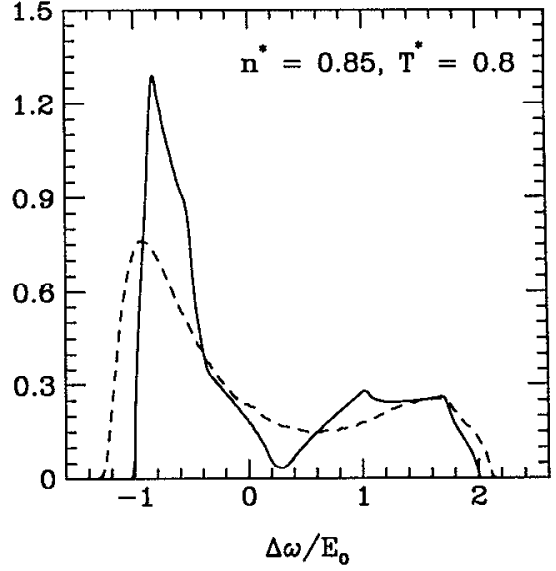

FIG. 7. The density of states $\bar{\rho}_{d}(\omega)$ for an idcal fec solid (solid) compared to the density of states of a liquid (dashed).

verse) excitons have a blue (red) shift of $2 E_{0}\left(E_{0}\right)$. Only the transverse excitons are spectroscopically active, since electromagnetic waves are transverse.

In Fig. 7 we plotted the density of states for a fcc solid, obtained using the expressions given in Refs. 21 and 22, and compare it to the simulation results of Fig. 1 for a $\mathrm{LJ}$ liquid. The qualitative agreement is quite remarkable. We clearly see the transverse and longitudinal modes in both calculations. It seems reasonable to adopt the fcc solid as a zero order reference and to develop a perturbation theory to hopefully model the dielectric function of a liquid. The idea of comparing a liquid to a solid is not new, and it has been applied to dielectric fluctuations by Stratt. ${ }^{23}$

In Figs. 8 and 9 we compare the density of states and absorption of a liquid with a disordered solid where the particles have a Gaussian distribution around their fec lattice positions, as in the Einstein solid. These simulations were performed in a way similar to the liquid simulations, but now the positions of the particles were generated by

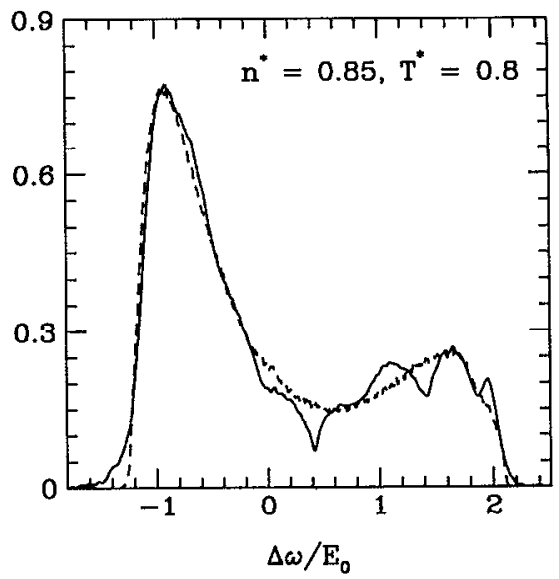

FIG. 8. The density of states $\vec{\rho}_{D}(\omega)$ for a Gaussian (Einstein) solid with standard deviation $0.09 \sigma_{\mathrm{LJ}}$ (solid) compared to the density of states of a liquid (dashed). The wiggles in the fcc simulation are finite size effects. 


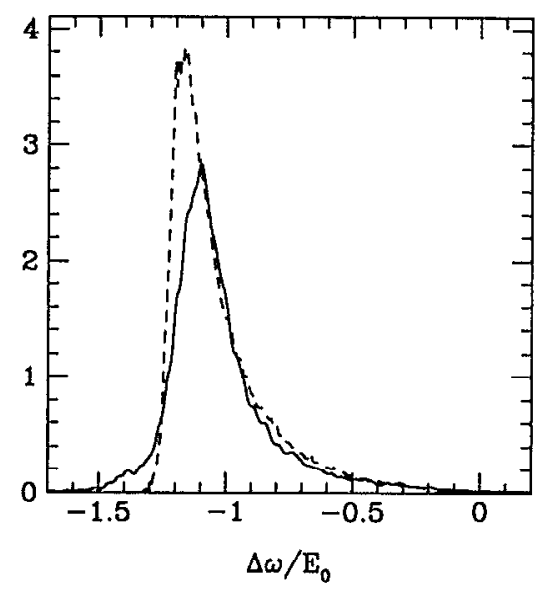

FIG. 9. Same as Fig. 8, but for the absorption spectrum $\epsilon^{\prime \prime}(\omega)$.

taking the fcc positions and adding a random number drawn from a Gaussian ensemble. We used the value of the Lindemann parameter ${ }^{24}$ the mean square displacement, as a fitting parameter. Matching the spectra we found that its fitting value essentially equals the Lindemann criterium for melting, the mean square displacement averages to $10 \%$ of the nearest-neighbor distance.

We next develop a simplified approximation for the dielectric function which illustrates that a perturbation from the solid is reasonable. We are interested in the polarizability

$$
\alpha^{\prime \prime}(\omega)=\lim _{k \rightarrow 0} \frac{\mu^{2}}{\pi} \operatorname{Im}\langle k|G(\omega)| k\rangle,
$$

and the density of states

$$
\rho(\omega)=\sum_{k} \frac{1}{\pi} \operatorname{Im}\langle k|G(\omega)| k\rangle,
$$

where

$$
G(\omega)=\frac{1}{\omega-H},
$$

and

$$
H=H_{\text {lattice }}+\Delta \text {. }
$$

In terms of the eigenfunctions and eigenvalues of a random $N \times N$ matrix we have

$$
\begin{aligned}
\alpha^{\prime \prime}(\omega) & =\frac{1}{\pi} \operatorname{Im}\langle k=0|G(\omega)| k=0\rangle \\
& =\frac{1}{\pi} \operatorname{Im} \sum_{\lambda} \frac{\left|\left\langle\psi_{\lambda} \mid k=0\right\rangle\right|^{2}}{\omega-E_{\lambda}-i \epsilon} .
\end{aligned}
$$

In the simulations presented in Figs. 8 and 9 we have used a numerical diagonalization.

We now propose a simplified scheme which captures the essential physics of the system. We shall approximate $\alpha^{\prime \prime}(\omega)$ by

$$
\alpha^{\prime \prime}(\omega) \simeq \frac{1}{\pi} \operatorname{Im} \sum_{\lambda} \frac{\left|\left\langle\psi_{\lambda}^{(2)} \mid k=0\right\rangle\right|^{2}}{\omega-E_{\lambda}^{(2)}+i \epsilon},
$$

where $\psi^{(2)}$ and $E^{(2)}$ are the eigenfunctions and eigenvalues of the $2 \times 2$ matrix

$$
\left(\begin{array}{cc}
E(k=0) & \tau\langle k=0|\Delta| k\rangle \\
\tau\langle k|\Delta| k=0\rangle & E(k)
\end{array}\right) .
$$

The parameter $\tau$ is taken to be a random variable, and $E(k)$ is the energy of the state perturbing the $k=0$ state. The approximation at this point is that only one state perturbs rather than many. This is an ingredient to the extension to many perturbing states described below Eq. (44). While the $2 \times 2$ approximation is clearly an oversimplification, it has the following attractive features: (i) It produces tractable results, (ii) For large $\Delta \omega=\omega-E_{0}$ it predicts

$$
\alpha(\omega) \propto \frac{\rho(\omega)}{\Delta \omega^{2}},
$$

which is a feature observed in a number of simulations. ${ }^{10}$ It should be stressed that this $\Delta \omega^{-2}$ dependence has a completely different origin than the gas phase tail. ${ }^{17}$ Here it follows as a property of perturbation theory, while in the gas phase it crucially depends on the $r^{-3}$ nature of the dipole-dipole interaction. Finally (iii) if $E(k)=E(0)$ and $\Delta$ is Gaussian, the $2 \times 2$ approach predicts a Gaussian line shape, in accordance with conventional pictures.

We now propose an improved approximation, taking into account that many small fluctuations contribute to the final line shape. For notational convenience we set $E(k=0)=0, \tau\langle k|\Delta| k=0\rangle=J$, and $E(k)=E$. The $2 \times 2$ matrix

$$
\left(\begin{array}{ll}
0 & J \\
J^{*} & E
\end{array}\right)
$$

has the eigenvalues

$$
E_{ \pm}=\frac{E}{2} \pm\left[\left(\frac{E}{2}\right)^{2}+J^{2}\right]^{1 / 2},
$$

and eigenfunctions

$$
\psi_{ \pm}=\left(1+\chi_{ \pm}^{2}\right)^{-1 / 2}\left(\begin{array}{c}
1 \\
\chi_{ \pm}
\end{array}\right)
$$

with

$$
\chi_{ \pm} \equiv E_{ \pm} / J
$$

We assume that $J$ is a random variable with Gaussian distribution and standard deviation $\sigma, P(J)$ $=\left(2 \pi \sigma^{2}\right)^{-1 / 2} \exp \left(-J^{2} / 2 \sigma^{2}\right)$. Using this we find for the polarizability $\alpha$,

$$
\alpha_{2}(\omega)=f(\omega, 0),
$$

and for the dielectric function

$$
\epsilon_{2}(\omega)=f\left(\omega,-E_{0}\right),
$$

where the subscript 2 reminds us that these correspond to a 2 level model, and 


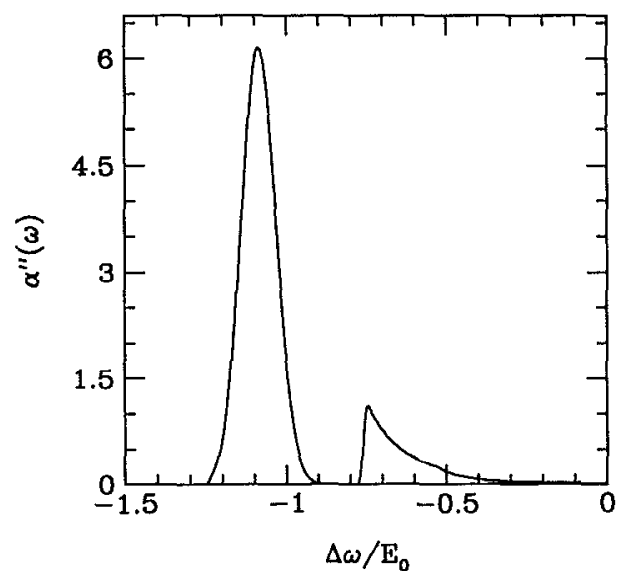

FIG. 10. The polarizability of a two level model $\vec{\alpha}_{2}^{\prime \prime}$, Eq. (41) with $\sigma^{2}=E_{0}^{2}$. The dip is unphysical and signifies the absence of third particle effects.

$$
\begin{aligned}
f\left(\omega, E_{1}\right)= & \int d J P(J) \int d E \rho_{s}(E) \\
& \times\left[\frac{1}{2}+s(\Delta \omega) \frac{\Delta E}{4}\left(\frac{\Delta E^{2}}{4}+J^{2}\right)^{-1 / 2}\right]^{-1} \\
& \times\left(1+\left\{\frac{\Delta E}{2 J}+s(\Delta \omega)\left[1+\left(\frac{\Delta E}{2 J}\right)^{2}\right]^{1 / 2}\right)^{2},\right.
\end{aligned}
$$

where $\Delta \omega=\omega-E_{1}, \rho_{s}$ is the density of states for the reference solid, $\Delta E=E-E_{1}=\Delta \omega-J^{2} / \Delta \omega$, and $s(\Delta \omega)$ is the sign function, $s(\Delta \omega)=1$ if $\Delta \omega>0$, and $s(\Delta \omega)=-1$ if $\Delta \omega<0$.

The density of states is given by

$$
\rho_{2}(\omega)=\int d E_{1} \rho_{s}\left(E_{1}\right) f\left(\omega, E_{1}\right)
$$

As is shown in Fig. 10, $\bar{\alpha}_{2}^{\prime \prime}$ has the same unphysical dip at $E_{0}$ as the gas phase pair atom approximation. ${ }^{17}$ We propose an improvement similar to the gas phase. We assume that many small, independent perturbations exist, adding up to the total perturbation, that is,

$$
\begin{aligned}
C(t) & =e^{-i \Omega t}\left\langle e^{i J t}\right\rangle \simeq e^{-i \Omega t}\left\langle e^{i J_{12} t}\right\rangle\left\langle e^{i J_{13} t}\right\rangle \cdots \\
& =e^{-i \Omega t}\left[C_{2, \sigma}(t)\right]^{N},
\end{aligned}
$$

where we keep $\sigma^{2} N$ constant. To obtain actual results we Fourier transformed Eq. (41), took the $N$ th power and transformed back. For $\sigma^{2} N=1 \cdot(n J)^{2}$, and $N$ large we obtained Figs. 11 and 12 for the density of states and $\epsilon$.

\section{EFFECTS OF NUCLEAR MOTIONS}

We now consider the effects of nuclear motion. The nuclear motion will be treated classically. We then have a semiclassical procedure where the electronic degrees of freedom are quantum mechanical and the nuclei are classical.

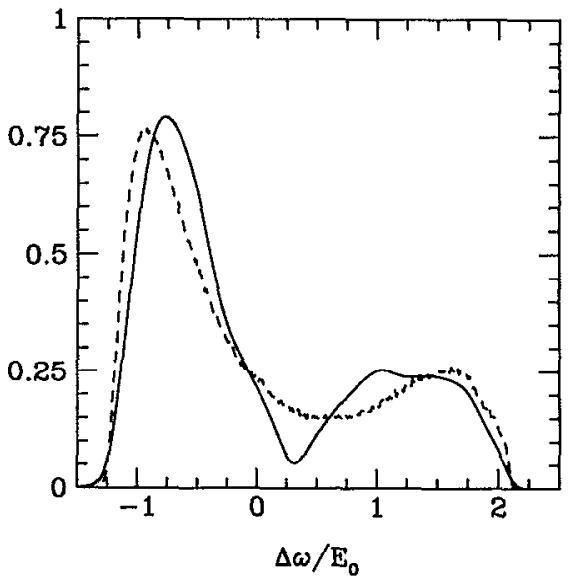

FIG. 11. The density of states obtained from Eq. (45) with $C$ following from $\mathrm{Eq}$. (44) (solid) compared to simulation results for a liquid (dashed).

The positions and velocities of the atoms are assumed to follow classical equations of motion

$$
\begin{aligned}
& \frac{d}{d t} r_{n}(t)=v_{n}, \\
& \frac{d}{d t} v_{n}(t)=F_{n}, \\
& F_{n}=-\sum_{m} \nabla_{n} V\left(r_{n m}\right),
\end{aligned}
$$

where $V(r)$ is the ground state interaction for which we use the Lennard-Jones potential.

We now approximate

$$
\exp _{+}\left[-i \int_{0}^{t} d \tau \hat{J}(\tau)\right] \simeq \exp \left[-i \int_{0}^{t} d \tau \hat{J}(\tau)\right]
$$

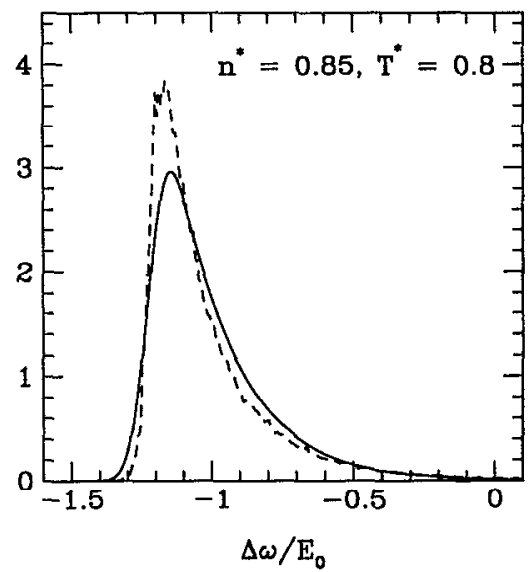

FIG. 12. The absorption spectrum $\epsilon^{\prime \prime}(\omega)$ following from Eq. (45) (solid) compared to simulation results for a liquid (dashed) at the same state point as Fig. 11 . 
so we replace the time ordered exponential by an ordinary exponential. This is in fact the lowest order approximation in an adiabatic expansion, ${ }^{25}$ where we assume that the $\hat{J}(t)$ at different times commute

$$
[J(t=0), J(t)]=0 .
$$

This semiclassical assumption enables us to make considerable progress. It is here that the matrix nature of $J$ shows up. For low density it is a good approximation, as then only binary interactions (presumably) are relevant and then Eq. (48) is always satisfied. It should hold for short times as corrections are of higher order in time.

For short times the particles do not move over large distances and we can make a gradient expansion of $J$, so that

$$
\hat{J}(t)=\hat{J}(0)+\Delta \hat{J}(t)
$$

with

$$
\Delta \hat{J}(t)=\sum_{n} \int_{0}^{t} d \tau v_{n}(\tau) \nabla_{n} \hat{J}(0) .
$$

We then have

$$
C(t)=e^{-i n t} C_{s}(t) C_{d}(t),
$$

where $C_{s}$ is an $N \times N$ matrix describing the static broadening

$$
C_{s}=\exp [-i \hat{J}(0) t],
$$

and the dynamics is described by the $N \times N$ matrix $C_{d}$,

$$
C_{d}(t)=\exp \left[i \int_{0}^{t} d t_{1} \int_{0}^{t_{1}} d \tau \sum_{n i} v_{n i}(\tau) \nabla_{n i} \hat{J}(0)\right] .
$$

To the second order cumulant expansion we obtain

$$
\begin{aligned}
C_{d}(t)= & \exp \left[-\int_{0}^{t} d t_{1} \int_{0}^{t_{1}} d \tau \int_{0}^{t} d t_{1}^{\prime} \int_{0}^{t_{1}^{\prime}} d \tau^{\prime}\right. \\
& \left.\times \sum_{n i} \sum_{n^{\prime} j}\left\langle v_{n i}(\tau) v_{n^{\prime} j}\left(\tau^{\prime}\right)\right\rangle\left\langle\nabla_{n} \hat{J}(0) \nabla_{n^{\prime} j} \hat{J}(0)\right\rangle\right] .
\end{aligned}
$$

Performing the thermal velocity average we find for short times

$$
\exp \left[i \sum_{n} v_{n} \frac{t_{1}^{2}}{2} \nabla_{n} \hat{J}(0)\right]=\exp \left(-\frac{k_{B} T}{m} \frac{t^{4}}{8} \hat{\lambda}_{p, p^{\prime}}\right),
$$

where exp denotes the exponent of the matrix $\hat{\lambda}_{p, p^{\prime}}$. We have

$$
\hat{\lambda}_{p, p^{\prime}}=\sum_{n i, p^{\prime \prime}} \nabla_{n i} \hat{J}_{p, p^{\prime \prime}} \nabla_{n i} \hat{J}_{p^{\prime \prime}, p^{\prime}},
$$

where $p$ runs over a basis of the optical degrees of freedom that may be different from $(n i)$. Since, by assumption, $\hat{J}(0)$ and $\Delta \hat{J}$ commute, both commute with $\hat{\lambda}$. If we take a basis in which $\hat{J}(0)$ is diagonal, we need only to consider the diagonal elements $\lambda_{p, p}$. Explicitly in terms of the eigenfunctions $\psi_{n i}^{(p)}$,

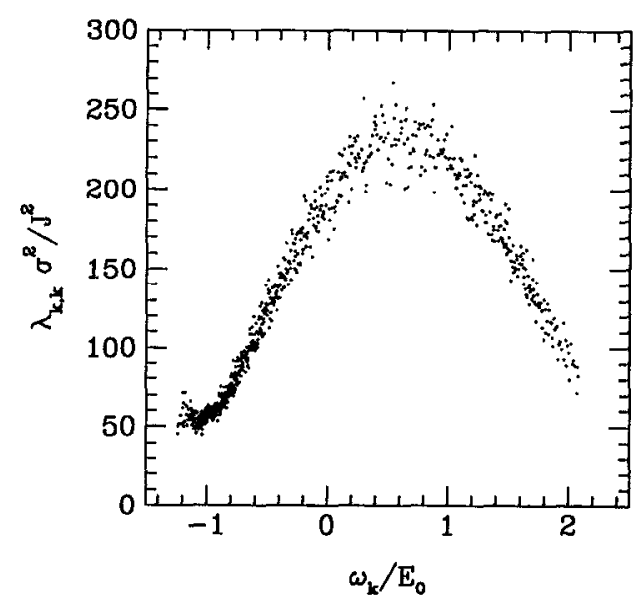

FIG. 13. The gradient squared of $J, \lambda_{p, p}[\mathrm{Eq} .(57)]$ as a function of the eigenvalue $\omega_{p}$ for the same system as in Fig. 1.

$$
\lambda_{p, p}=\sum_{n i j}\left|\sum_{j \gamma}\left(\nabla_{n i} T_{i j, j \gamma}\right) \psi_{j \gamma}^{(p)}\right|^{2} .
$$

The first conclusion is that motional corrections are proportional to $e^{-\kappa t^{4}}$, compared to a $e^{-a t^{2}}$ dependence which is typical for a static linewidth. Nuclear motions are therefore either irrelevant, or they dominate and the crossover between these two extremes is abrupt (varying, say, the mass).

We performed a molecular dynamics simulation in order to evaluate the gradients of $J$. A typical result for a liquid is given in Fig. 13. A remarkable feature is that the gradient seems to depend on the energy of the particular state. Also the gradient of $J$ seems to be inversely proportional to the density of states at that given energy. Even though the gradient squared varies considerably for the optically active frequency range, the $t^{4}$ dependence will ensure that for a reasonable estimate we can replace $\lambda_{p, p}$ by a number that is independent of $p$, considering Fig. 13 we take $\lambda_{p, p}=50 J^{2} / \sigma^{2}$ in the following discussion.

The most interesting question is how all of this works out for a real liquid such as argon. Argon can reasonably be described by a Lennard-Jones liquid with parameters $\epsilon_{\mathrm{LJ}}=119.8 \mathrm{~K}, \sigma_{\mathrm{LJ}}=0.3405 \mathrm{~nm}$. Furthermore the mass is 39.95 a.u. $=6.634 \times 10^{-26} \mathrm{~kg}$, the polarizability $\alpha=1.6411$ $\times 10^{-27} \mathrm{~m}^{3}$. We are here mainly interested in order of magnitude estimates, and for the isolated atom transition frequency $\Omega_{0}$ a reasonable number is $10 \mathrm{eV}$. For these numbers the electronic dephasing time (inverse linewidth) $t_{\mathrm{el}}=1 / J \simeq 5 \mathrm{fs}$. The gradient of the energies gives us a time scale after which the motion becomes important $t_{\text {mot }}$ $=\left(8 m / k_{B} T \lambda_{p, p}\right)^{1 / 4}$ and for argon this is $50 \mathrm{fs}$; the effect of the nuclear motion is therefore irrelevant for linear optical properties of liquids. For a gas, the nuclear motion gives rise to collisional broadening which is of comparable magnitude as the inhomogeneous broadening. ${ }^{17}$

The theory developed here points out that systems in which nuclear motion is important should be weakly interacting and made of light atoms. In order to observe the 
nuclear motion in an optical experiment one has to go to nonlinear experiments, such as the photon echo. ${ }^{26,27}$ Our theory suggests a $e^{-\kappa t^{4}}$ decay rather than the simple exponential decay which is a characteristic of conventional homogeneous broadening. This should be observable in a photon echo experiment.

In the present discussion we have made the semiclassical approximation Eq. (48), which is fundamental to our treatment, the gradient expansion and the short time straight line approximation. The last approximation is justified for liquid argon; $t_{\text {mot }}$ is quite a bit shorter than the mean free time, which is $\sim 0.2 \mathrm{ps}$. The straight line approximation can be easily improved, which may be useful for systems that are interacting more weakly than argon. We make a cumulant expansion of the last "exp" of Eq. (51). The first cumulant is proportional to $\Delta r$ and is zero by symmetry. The second cumulant is proportional to $\Delta r_{n} \Delta r_{m}$. This cumulant is a complex many body quantity and evaluation of its dynamics is hard. To estimate it we keep the diagonal, $i=j$ contribution. In terms of the velocity autocorrelation function $\rho(t)=\left\langle v_{n} v_{n}(t)\right\rangle$ we then find

$$
\begin{aligned}
& \exp \left[\int_{0}^{t} d t_{1} \sum_{n i} \Delta r_{n i} t_{1} \nabla_{n t} J(0)\right] \\
& \quad=\exp \left[-\frac{1}{2} \hat{\lambda}_{p, p^{\prime}} \int_{0}^{\infty} d t_{1} \rho\left(t_{1}\right)\left(\frac{2}{3} t^{3}-t_{1} t^{2}+\frac{1}{3} t_{1}^{3}\right)\right] .
\end{aligned}
$$

For short times the integral over $t_{1}$ is proportional to $k_{B} T t^{4} / 4 m$, for times longer than the velocity correlation time it crosses over to a $2 D t^{3} / 3$ dependence, where $D$ is the self-diffusion constant. The self-diffusion constant appears here due to the fact that we retained the $i=j$ contribution. The $t^{3}$ dependence we believe to be generic, that is independent of the diagonal approximation.

Recently a number of articles have appeared that proposed to use a phonon description for the dynamics of liquids. ${ }^{28,29}$ In this approach, which is rigorous for short times, phonons are defined as "instantaneous" normal modes. While we have shown in this paper that the solid is a good reference for the optical properties, this does not necessarily prove the usefulness of a solid reference for the nuclear dynamics. For one thing in order for the instantaneous normal mode picture to be meaningful, the modes have to be sufficiently long lived (at least a few vibrational periods). Whether this is the case is still an open and important question. Alternatively it is possible to formulate a theory that incorporates nuclear dynamics through collisions. A careful analysis shows that even for liquids it is very reasonable to talk about collisions. ${ }^{30}$ This approach has many advantages, it is consistent, and it produces very reasonable nontrivial predictions.

\section{ACKNOWLEDGMENTS}

The support of the Air Force Office of Scientific Research, the National Science Foundation, and the Center for Photoinduced Charge Transfer is gratefully acknowledged. We thank Ningjun Wang for providing a computer program to calculate the MSA results.

${ }^{1}$ N. Schwentner, E.-E. Koch, and J. Jortner, Electronic Excitations in Condensed Rare Gases (Springer, Berlin, 1985).

${ }^{2}$ J. Wörmer, M. Joppien, G. Zimmerer, and T. Möller, Phys. Rev. Lett. 67, 2053 (1991).

${ }^{3}$ L. R. Pratt, Mol. Phys. 40, 347 (1980); J. S. Høye and G. Stell, J. Chem. Phys. 73, 461 (1980); 75, 5133 (1981).

${ }^{4}$ D. Chandler, K. S. Schweizer, and P. G. Wolynes, Phys. Rev. Lett. 49, 1100 (1982).

${ }^{5}$ M. J. Thompson, K. S. Schweizer, and D. Chandler, J. Chem. Phys. 76, 1128 (1982).

${ }^{6}$ Y.-C. Chen, J. L. Lebowitz, and P. Nielaba, J. Chem. Phys. 91, 340 (1989).

${ }^{7}$ B. Cichocki and B. U. Felderhof, J. Chem. Phys. 90, 4960 (1989).

${ }^{8}$ I. Messing, B. Raz, and J. Jortner, J. Chem. Phys. 66, 2239 (1977).

${ }^{9}$ H. M. Sevian and J. L. Skinner, J. Chem. Phys. 97, 8 (1992).

${ }^{10}$ H. Fidder, J. Knoester, and D. Wiersma, J. Chem. Phys. 95, 7880 (1991).

${ }^{11}$ R. F. Loring, J. Chem. Phys. 92, 1598 (1990); A. M. Walsh and R. F. Loring, Chem. Phys. Lett. 186, 77 (1991); R. F. Loring, J. Chem. Phys. 94, 513 (1990); N. E. Shemetulskis and R. F. Loring, ibid. 95, 4756 (1991).

${ }^{12}$ K. Ganguly and R. M. Stratt, J. Chem. Phys. 95, 4418 (1991); 97, 1980 (1992); Z. Chen and R. M. Stratt, ibid. 97, 5687 (1992); 97, 5696 (1992).

${ }^{13}$ M. D. Winn and D. E. Logan, J. Chem. Phys. 96, 4818 (1992).

${ }^{14}$ M. S. Wertheim, J. Chem. Phys. 55, 4291 (1971).

${ }^{15} \mathrm{P}$. Wolynes, J. Chem. Phys. 96, 5133 (1987).

${ }^{16}$ Z. Chen and R. M. Stratt, J. Chem. Phys. 95, 2669 (1991); R. M. Stratt and B. C. Xu, Phys. Rev. Lett. 62, 1675 (1989); B. C. Xu and R. M. Stratt, J. Chem. Phys. 91, 5613 (1989).

${ }^{17}$ J. A. Leegwater and S. Mukamel, Phys. Rev. A (submitted).

${ }^{18}$ Sec, e.g., S. W. Dc Leeuw, J. W. Pcrram, and E. R. Smith, Proc. R. Soc. London, Ser. A 373, 27 (1980).

${ }^{19}$ M. L. Mehta, Random Matrix Theory, 2nd ed. (Academic, Boston, 1991 ).

${ }^{20}$ A. S. Davydov, Theory of Molecular Excitons (Plenum, New York, 1971).

${ }^{21}$ M. H. Cohen and F. Keffer, Phys. Rev. 99, 1128 (1955).

${ }^{22}$ N. Wang, J. A. Leegwater, and S. Mukamel (unpublished).

${ }^{23}$ B.-C. Xu and R. M. Stratt, J. Chem. Phys. 91, 5613 (1989).

${ }^{24}$ F. A. Lindemann, Z. Phys. 11, 609 (1910).

${ }^{25}$ R. M. Wilcox, J. Math. Phys. 8, 962 (1967).

${ }^{26}$ J. A. Leegwater, L. E. Fried, and S. Mukamel, Z. Phys. D (in press, 1993).

${ }^{27}$ Y. Yan and S. Mukamel, J. Chem. Phys. 94, 179 (1991).

${ }^{28}$ G. Seeley and T. Keyes, J. Chem. Phys. 91, 5581 (1989); B.-C. Xu and R. M. Stratt, ibid. 92, 1923 (1990).

${ }^{29}$ T.-M. Wu and R. Loring, J. Chem. Phys. 97, 8568 (1992).

${ }^{30}$ J. A. Leegwater, J. Chem. Phys. 94, 7402 (1991); 95, 8346 (1991). 